diseases has been diagnosed as Type - Classic of Hodgkin lymphoma (mixed celularity).

Results The treatment is done according to protocol for Hodgkin lymphona, with three cycles of OEPA (Prednisone, Vincristine, Doxorubicine, Etoposide), and three cycles of COPP (Prednisone, Procarbazine, Vincristine, Cyclophosphamide).After therapy, the PET - scan has been made and its results show that nowhere in the body is present malignant infiltration.

Conclusion OEPA and COPP are very efficient therapy in the treatment of Hodgkin lymphoma.

\section{CHEMOTERAPEUTIC TREATMENT OF RHABDOMYOSARCOMA BOTRYOIDE}

doi:10.1136/archdischild-2012-302724.0527

'F Selimi, 'V Grajqevci-Uka, 'R Macastena-Maxhuni, 'B Abrashi, ${ }^{2}$ V Hasbahta. 'HematoOncology Department; '2Gastroenterology Department, Pediatric Clinic, University Clinical Centre of Kosovo, Prishtina, Kosovo

Introductions Rhabdomyosarcoma (RMS) is malignant tumor which arises from embryonic muscle cells. RMS is the most common type of soft-tissue cancer in children. It can develop on children at any age, but it's most common in those between 2 and 6 years old and 15 and 19 years old.

Purpose Presentation of the case with RMS botryoides, which was presented to the Hematology/Oncology Unit at Pediatric Clinic and has received chemotherapy treatment.

Materials and Methods A female child, 2 years old, who was initially admitted in Clinic University Centre of Tirana, with presence the mass of tumor in vulvovaginal region. The tumor mass appeared after the birth and it has been increasing in size since then. At University Clinic Center of Tirana the patient has been operated, and the tumor has been removed from her. Detailed Histopathological analyses has shown that the removal part of her was botryoide Rhabdomyosarcoma. The diagnose of the patient was made based on anamnesis, clinical examination, laboratory tests, radiological images, bone marrow aspiratione, histopathological analysis. After the diagnoses it was indicated the treatment protocol of chemotherapy for extracranial rhabdomyosarcoma VAC.

Conclusion The total Resection of the tumor mass, the application of the protocol for RMS botryoide, supportive care, monitoration of the chemotherapy toxicity has resulted in absence of minimal residual disease, which is confirmed by the follow up of her clinical status, laboratory tests, radiology tests, that has resulted the absence of secondary deposits.

\section{IATROGENIC TRACHEAL RUPTURE IN A CHILD: CASE STUDY AND REVIEW OF LITERATURE}

doi:10.1136/archdischild-2012-302724.0528

${ }^{1} \mathrm{MS}$ Paksu, ${ }^{2} \mathrm{AA}$ Kilinc, ${ }^{1} \mathrm{~N}$ Asilioglu, ${ }^{3} \mathrm{M}$ Gunaydin, ${ }^{2} \mathrm{~T}$ Aydin, ${ }^{4} \mathrm{~A}$ Guzel, ${ }^{5} \mathrm{M}$ Ceyhan ${ }^{1}$ Ondokuz Mayis University Faculty of Medicine, Pediatric Intensive Care Unit; ${ }^{2}$ Ondokuz Mayis University Faculty of Medicine Department of Pediatrics; ${ }^{3}$ Ondokuz Mayis University Faculty of Medicine Department of Pediatric Surgery; ${ }^{4}$ Ondokuz Mayis University Faculty of Medicine Department of Pediatric Emergency; ${ }^{5}$ Ondokuz Mayis University Faculty of Medicine Department of Radiology, Samsun, Turkey

Tracheal rupture is rare in childhood and optimal treatment is not clear. A 14-year old boy was admitted to a local hospital after sudden loss of consciousness. He underwent endotracheal intubation and was referred to our hospital. The patient's history revealed that he had voluntarily inhaled butane gas. The physical examination was consistent with coma and cardiogenic shock, and the chest $\mathrm{x}$-ray showed pulmonary edema. The patient was admitted to the intensive care unit, and diuretic and inotropic therapy was started. In the third hour of monitoring of the patient under mechanical ventilation, subcutaneous emphysema and pneumothorax at the right hemithorax were observed without deterioration of the vital functions. Thoracic computed tomography (CT) scan findings were consistent with tracheal rupture. The patient was monitored conservatively without surgery. On the fifth day of hospitalization, his tube was removed, and he was discharged on the twelfth day with a positive prognosis. In this study, a tracheal rupture case after endotracheal intubation is presented in which the patient recovered completely with conservative therapy.

\section{OSTEOGENISIS IMPERFECTA IN A DEFINED BIRTH COHORT: REGIONAL EXPERIENCE AND THE NEED FOR A NATIONAL PLAN}

doi:10.1136/archdischild-2012-302724.0529

S Oadri. Paediatrics, University Hospital Limerick, Limerick, Ireland

Background and Aims We aim to analyse the clinical characteristics of OI within a 10 year regional birth cohort in Ireland and to highlight the management challenges and the need for a National level referral and treatment structure.

Methods Case notes of all patients with OI from 2002 to 2011 were reviewed for clinical characteristics, radiological findings, results of molecular genetics and the comprehensive management including treatment received abroad.

\section{Results}

Case1: Female type III OI born by caesarean section at term noticed to have several fractures in-utero and infancy; now 10 years with short stature \& respiratory problems.

Case2: Male type IV OI, born by CS at term. Multiple antenatal fractures noted on postnatal skeletal survey. Presently 6 years old.

Case3: Male type III OI, presented at birth with multiple fractures, and significant ligamentous laxity. Presently 4 years old.

Case4: Female type V OI, born by CS at term. Diagnosed at 9 weeks when presented with fractures. Developed subsequent crush fractures of vertebrae despite being on pamidronate infusions; presently 3 years old.

Case5: Female type IV OI, born by CS at term with antenatal suspicion of skeletal dysplasia. Presently 2 years old with associated developmental delay.

Case6: Male type IV OI, followed up since 27 weeks gestation and intrauterine death following the development of non-immune hydrops at 34 wks.

Conclusion As more children are surviving nationally there is a need for a single specialised and comprehensive paediatric metabolic bone diseases unit providing a structured quality of care and avoiding travel abroad.

\section{ACQUIRED MICROCEPHALY IN A CHILD WITH ABSENT SPEECH: HOW TO STUDY?}

doi:10.1136/archdischild-2012-302724.0530

J Sales Marques, D Rocha, Al Pinto Pais. Centro Hospitalar Vila Nova de Gaia/Espinho EPE, Vila Nova de Gaia, Portugal

Introduction Microcephaly is defined as reduce of the head circumference less than 2 SDS for age, sex and gestation.

Purpose Three different cases of acquired microcephaly and absent speech are presented with the purpose to find the better form to study patients with this clinical association.

\section{Results}

Case 1 - Eight-year old girl, has acquired microcephaly since 15 months of age, absent speech, delay motor skills, seizures and angelman-like features. Chromosome 15 study confirmed Angelman Syndrome. 